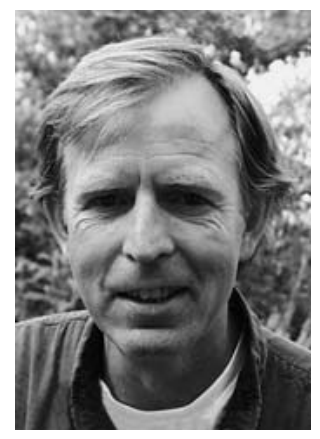

\title{
Quantum Case Analysis
}

Do you remember your first computer? Mine was a Mac Plus that possessed a random access memory (RAM) of 1 MB! In 1987 this was enough to run the first version of MacRepertory; now not enough to save one snapshot! In 25 years the technical abilities of computers have skyrocketed, and the impact of new developments in the coming 25 years can only be imagined.

For homeopathy the video and the computer have had huge influence. Video cases improved the quality of teaching tremendously. Who can better teach you a remedy than a patient telling you about his state? Earlier we could read or hear about a remedy, but now we could, as it were, taste it. A huge difference similar to reading about a red fruit the size of a chestnut, with tiny seeds on its surface and then getting the opportunity to taste the real thing - a strawberry. A complete library of homeopathy books can now be on your hard disk, and what is more, you can search in it at an amazing speed. What will be available to us in 2037?

A development that may open incredible new vistas, also for homeopathy, has been set in motion by Dutch nanoscientist Leo Kouwenhoven. He caused great excitement among scientists with research published in Science in April 2012.

In the 1930s, the brilliant Italian physicist Ettore Majorana deduced from quantum theory the possibility of the existence of a very special particle, a particle that is its own anti-particle. That Majorana fermion would be right on the border between matter and anti-matter. Leo Kouwenhoven's research group managed to create a nanoscale electronic device in which a pair of Majorana fermions 'appear' at either end of a nanowire. The discovery of Majorana fermions opens up a new and uncharted chapter of fundamental physics; they may also play a role in cosmology, as a proposed theory assumes that the mysterious 'dark matter', which forms the greatest part of the universe, is composed of Majorana fermions.

Furthermore, scientists view the particles as fundamental building blocks for the quantum computer. Such a computer is far more powerful than the best supercomputer because it has the ability to be in more than one state simultaneously. Whereas digital computers require data to be encoded into binary digits (bits), quantum computation utilizes quantum properties to represent data and perform operations on these data. So far quantum computers have only existed in theory, now they may soon become a reality.

In 2037 your quantum computer might record everything your patient says while simultaneously analyzing the information and comparing it with a huge memory of provings, materia medica and cured cases. By the time the interview is over and 3-D photos of the patient, DNA and all sorts of physical parameters have been entered into the equation, a differential diagnosis of homeopathic substances is proposed together with a calculation of the likelihood of a substance being the simillimum. This seems a possible scenario. Whether it would actually still involve you or whether the patient would simply run an app at home - who knows? You and homeopathy may have moved on to completely new ways of determining, creating and applying the simillimum.

Practically all theoretic particles that are predicted by quantum theory have been found in the last decades, with just a few exceptions, including the enigmatic Majorana particle and the well-known Higgs boson. But Ettore Majorana the person is every bit as mysterious as the particle. In 1938 he withdrew all his money and disappeared during a boat trip from Palermo to Naples. Whether he killed himself, was murdered or lived on under a different identity is still not known. You don't withdraw your money and then kill yourself, do you? But then he did write what seems to be a suicide note. Was he killed by the Nazis because he refused to be involved in developing a nuclear weapon, or because they feared he might do so for the allies? Was he indeed seen in Argentina? No trace of Majorana was ever found. As if he had merged with his own anti-particle. As if he changed roles with the mysterious dark matter the particle named after him possibly explains.

Could homeopathy offer an answer as to his state and possibly his fate? It's tempting to at least have a look at Origanum majorana. The remedy has suicidal disposition as one of its known symptoms. The Lamiaceae are ambitious and have a great need of acknowledgement. Ettore Majorana was extremely brilliant, which would be a good way to get recognition, but theoretical physics and the sphere of action for which Origanum is known don't really seem to compute. Also praise may not have been an important motivation for him as he left writing a paper about another discovery he made to James Chadwick who won the Nobel Prize for it.

We could analyse the books and films made about Ettore and speculate about his remedy. But we may better wait until 2037, then feed all personal writings by Ettore Majorana, his DNA and his photo into the quantum computer that he should posthumously receive a Nobel Prize for, and accept the - also posthumously - suggested remedy as his most likely simillimum.

Harry van der Zee 